\title{
Consent — a study from the dental patients' viewpoint
}

Consent: the patients' view - a summary of findings from a study of patients' perceptions of their consent to dental care by J. King Br Dent J 2001; 191: 36-40

\section{Aim}

A study was carried out which aimed to investigate peoples' perceptions of how their consent was given for dental treatment.

\section{Method}

A structured questionnaire was completed by 50 patients receiving treatment in the British NHS and 12 of them completed an in-depth recorded interview. The data was analysed using a combination of quantitative and qualitative methods.

\section{Results}

The findings showed that $80 \%$ of patients had not been given any written treatment plan (FP17DC). $79 \%$ of patients mistakenly thought that the form signed requesting NHS treatment (FP17) was a consent form. When options were offered patients were more likely to consider that they were involved in the consenting process. However consent is still often implied rather than explicit. There was a range of experience reported by patients from those who felt that the dentist made the treatment decisions to those who felt that decisions had been made collaboratively. Although some patients were happy with the way their consent was obtained examples were also given of lack of information, confusion and even of deceit.

\section{Conclusions}

There is an urgent need to clarify the status of NHS documentation regarding consent and a general need for awareness to be raised in the dental profession about the importance of obtaining consent which is freely given based on appropriate information which has been adequately understood.

\section{In Brief}

- Raises awareness of the importance of consent procedures for patients in everyday practice

- Highlights people's awareness of the time constraints of the NHS fee structures

- Draws attention to the confusion about NHS documentation that patients experience

\section{Comment}

$\mathrm{T}$ here has been much written on the question of consent and how and what is needed to be done to ensure that the patient has agreed to treatment. Sometimes that process can be described as nothing more than an elaborate ritual, a ritual designed to satisfy the known requirements of consent, a ritual undertaken without thought as to its real purpose. This paper exposes that concept. One of the last sentences of this paper suggests that 'moral and legal considerations, as well as the demands of good practice, call for consent to be taken to taken very seriously by dentists and patients alike'. That is true. But surely the essential elements of the consenting process itself are embraced by good practice?

Good practice would fulfil the moral and legal requirements and more. Take for example questions of treatment costs and time. This study shows that over $1 / 3$ of patients received no information on these matters. The only analogy I can make is to suggest how discomforting it is to take your car in for service in the hope that it will be ready that evening and wondering how much the bill will be. Purists may regard such information as essential elements of legal and moral consent that is debatable. They are certainly essential to good practice through common courtesy.

This paper tells us what the patients feel about the 'ritual' by telling their story through their comments at interview. On the whole it shows that these patients were happy with the information given to them and the manner in which it was given. Indeed, there is some sympathy for the pressures under which NHS practitioners work! However it is clear that there is considerable room for improvement, and that that improvement need not necessarily be limited to the general dental services. Some of the comments and the findings of this research would apply equally to the provision of dental care in the hospital and community services. Perhaps, in particular, the provision of written information about treatment especially when patients are undergoing treatment over many visits. Such written information should not, of course, be a substitute for patient dialogue but merely an adjunct to the process of obtaining agreement. If you have read this commentary to this point then I hope I will have persuaded you to look at the whole article. It is well written and worth the read, no matter what your area of practice.

Andrew M Bridgman

University of Manchester Dental Hospital 